\title{
Usage of Implant Contraceptive Method by Sexually Active Women: An Exploration of The Factors Contributing To Implant Contraceptive Method Uptake in Vhembe District, Limpopo Province, South Africa.
}

Rhulani Caroline Shihundla

University of Venda

Rachel Tsakani Lebese ( $\nabla$ rachel.lebese@univen.ac.za)

University of Venda School of Health Sciences https://orcid.org/0000-0002-3209-5660

Sonto Maria Maputle

University of Venda

Research

Keywords: Implant contraceptive methods, Sexually active women, Uptake, Usage.

Posted Date: July 12th, 2021

DOI: https://doi.org/10.21203/rs.3.rs-641140/v1

License: (c) (i) This work is licensed under a Creative Commons Attribution 4.0 International License. Read Full License 


\section{Abstract \\ Background}

The estimation of unmet contraceptive needs triggered the National Department of Health in South Africa to introduced the Implant contraceptive method in 2014 to increase the range of contraceptive options for sexually active women. Implant contraceptive uptake remains low in the Vhembe District despite its effectiveness in preventing unintended pregnancy. Very little is known about the factors that affect the usage of this cost-effective device.

\section{Aim}

To explore sexually active women's knowledge, practices and attitudes related to the usage of the Implant contraceptive method in Vhembe District, Limpopo Province.

\section{Methods}

The study was qualitative, explorative, descriptive and contextual, conducted using a non-probability purposive technique to sample 62 sexually active women aged from 18-45 years. The sample size was determined by data saturation. Five (5) focus group discussions were conducted using semi-structured interviews, which lasted for 45-60 minutes. Tesch's eight steps of open coding were used to analyse data.

\section{Results}

Results revealed that sexually active women experience some challenges related to Implant Contraceptive Method. The majority of women had little knowledge about the device, making it difficult for them to use the method. The majority of sexually active women indicated that they are interested in the method and would like to enjoy the benefits.

\section{Conclusion}

Sexually active women's intentions to utilise the Implant contraceptive method was very low as the majority of them had a negative attitude towards the method, due to insufficient knowledge. Professional nurses should inform women and public members about newly introduced Implant contraceptive method at Primary Health Care facilities to enhance usage.

\section{Plain English Summary}

There are different types of contraceptive methods that sexually active women in different contexts use. They generally have their preferences influenced by the different environments that they live in and the social norms that they subscribe to. One of the methods available for women to use is the Implant though its uptake remains low in women of the Vhembe district. This study, therefore, sought to explore sexually active women's knowledge, practices and attitudes related to the usage of the Implant contraceptive method in Vhembe District, Limpopo Province. Focus group discussions were held to solicit views from the sexually active women on their knowledge, attitudes and practises towards the Implant as a contraceptive method. The study's findings showed women are interested in using this method as it offered more savings and lasted for a longer period. Some challenges were found to be contributing to the low uptake of this method. These were cited as lack of knowledge and the attitude that women had towards this method as they felt it causes bleeding, leading to reduced sexual pleasure. There is, therefore, a need for health service providers to embark on an intensive awareness campaign to market and improve the uptake of this contraceptive method.

\subsection{Background}

Knowledge is the ability of a person's mind to store learned information that one can apply practically, displaying the existing skills [1]. Sexually active women (SAW) 's knowledge about the Implant contraceptive method (ICM) refers to information they learned before, during, and after using contraceptives. Although long-acting reversible contraceptives (LARC) such as ICM is available in the United States (US) adolescents, use less effective methods, such as short-acting contraceptive methods (SACM) and barrier method like condoms [2].

This is confirmed by the conducted in the US by [2], who indicated that about 54\% of SAW who were using SACM or condoms had an abortion. Nevertheless, [3] found that SAW's knowledge about contraceptive methods (especially female-controlled methods) like ICM is higher in the US and Mexico. Health talks in facilities are mostly given to SAW while males are at work. 
Interviews conducted among SAW in Ethiopia displayed that the participants knew methods of contraception where $82 \%$ of SAW could identify at least one of the LARC methods [4]. Whereas, [3] in their study revealed that SAW did know the availability of modern contraceptives at facilities, but the use of such available methods was said to be hindered by gender roles and other religious objection. In Ghana, [5] identified two most widely known contraceptive methods: male condoms and injectables. The study conducted in Limpopo Province by [6] found that 60\% of adolescent mothers displayed knowledge of some contraceptives but very few used injectables and condoms

Worldwide the use of modern contraceptives has increased over the last period of ten years. Contrary to this report, about $25 \%$ of SAW who are supposed to be using contraceptives for child spacing were not utilising them during the study in Sub-Saharan Africa. That was said to be influenced by lack of information and misunderstanding attached to these contraceptives, and those are the main reasons for not using them for child spacing [7].

It has been found that the use of contraceptives is not only influenced by knowledge but there are other factors that motivate SAW to use them. In Ethiopia, SAW's knowledge of contraceptive methods was high; however, the contraceptive use prevalence rate was lower than expected [8]. Whilst in Mexico SAW believe that the use of contraceptives may lead one to ill health and infertility in the future. Thus, SAW using contraceptives were associated with being unfaithful to their sexual partners [5]. Furthermore, [6] added that even though SAW had information about available contraceptives, their male sexual partners dislike the use of contraceptive methods in such a manner that they are afraid to utilise them. This is because male partners had opposite information about these contraceptives; for example, they said it interfered with the feeling of satisfaction during intercourse.

Utilisation of contraceptives by SAW is also affected by how health professionals render these services. For SAW to know availability, utilisation and effective management of side effects of contraceptive methods, health professionals must inform them continuously at health care facilities. Failure of health providers in giving information tends to waste SAW s' time trying to find out on their own the methods that best suit them [9]. According to a study conducted by [10], the responsibility of health providers is to educate SAW about the different contraceptive methods, which can assist them in making an informed decision about the contraceptive method suitable for them.

It was further added that the foundation of accurate history taking is good communication between the professional nurses and SAW [11]. It is important to communicate with SAW because the method that appears 'ideal' in professional nurses' minds may not be acceptable to the SAW. The South African National Contraception Clinical Guidelines concurred that most SAW have some degree of knowledge, but limited knowledge about contraceptives leads to underutilisation of those methods [12].

The use of modern contraceptive methods remains low in Sub-Saharan Africa, thereby increasing the likelihood of unwanted pregnancy and disturbing life programmes of most SAW worldwide. These incidences are mostly linked with SAW's behaviours of failure to use LARC methods that have a lower failure rate for women to fall pregnant. The contributory factors for SAW to have unintended pregnancies may be due to personal beliefs, myths and insufficient insight about available LARC at the nearby PHC facilities [5].

In a study in the US and New Mexico, [13] identified that the use of LARC, the Intra-uterine copper device (IUCD) and ICM, is more common in other developed countries than in the US where the rate is increasing, although it rests at 7.7\%. In Pakistan, SAW have no insight into modern contraceptive methods because they do not use them; they still believe in not utilising modern contraceptive methods. Some SAW in Pakistan utilise traditional methods; tie a cloth, put it in the vagina, as it is believed to impede movement of sperms and prevent conception [8].

In Pakistan SAW clarified their fear of needles and pain during insertion and removal of the device as the other reason for not using ICM. In a study on the conversation about contraceptive services in Ghana, [14] found that students remarked that it is not easy to exchange sexual matters with partners and believed that contraceptives were for females only. Some of the participants had the feeling that contraceptives were not for poor people or that it is wrong to use contraceptives.

The utilisation of ICM among SAW was initially low in the European countries. However, the use of ICM rose from $0.5 \%$ to $3.4 \%$ within the period of six years from 2004 to 2010, following the information given to SAW about its availability and effectiveness [15; 16]. On the other hand, a study conducted in Africa by [4] argues that irrespective of the information given to SAW about the effectiveness and availability of LARC, the use of ICM remains very low in Zambia.

The perceived factors that make ICM lower than expected are the deficient information received by SAW about the availability, effectiveness, and ineffective management of side effects. In addition, negative attitudes by SAW towards the ICM, fear of side effects, lack of partner support, medical-related challenges, unavailability of methods, desire to have more children, age, and preference of SACM. Health providers have to give truthful information regarding ICM $[17 ; 18 ; 19]$.

Similarly, studies in Kenya and Nigeria revealed misconceptions, side effects, and lack of support by sexual partners were the reasons for the discontinuation of ICM utilisation [20]. In South Africa, [9] reported low utilisation of ICM due to marked side effects especially irregular bleeding tendencies. [21] Found that in South Africa, there was a marked decline following the initial high usage of the newly introduced ICM in the two

Page 3/12 
provinces, Gauteng and North West, where their studies were conducted. The main reason for that considerable decline was said to be frequent side effects.

In Limpopo Province, however, there were no studies found on the use of ICM. Besides the availability of enough ICM supplies at PHC facilities in Limpopo, there was underutilisation of ICM [22]. From February 2014 to April 2017, 436,016 SAW aged 18-45 years attended reproductive health services in the two Sub-Districts, Thulamela and Musina, in Vhembe District of Limpopo Province. However, only 7,964 were on ICM, and the majority were found to be using SACM [23].

The implant contraceptive method (ICM) was presented as a cost-effective and highly reliable form of LARC that was more widely accessible to all SAW considering that it will prevent unintended pregnancy [24]. However, regardless of the effectiveness and availability of ICM, in Vhembe District, unintended pregnancies were still high, as evidenced by the rate of abortion recorded between 2014/2017 at 35\% in Vhembe District [23]. Through experience and research findings, it was revealed that the prevalence of teenage pregnancy and termination of pregnancy (TOP) was high, which tend to be risky for the victims. These unintended teenage pregnancies progressed from $7.0 \%$ in 2014 to $7.9 \%$ in 2017 . It was discovered that after the introduction of ICM, the uptake has been low compared to SACM. That was confirmed in all PHC facilities of Thulamela and Musina Sub-Districts during data review meetings with facilities operational managers (OMN)

Despite its effectiveness in the prevention of unintended pregnancy, ICM does not protect against sexually transmitted infections (STIs), including Human Immunodeficiency Virus/Acquired Immunodeficiency Syndrome (HIV/AIDS); the use of condoms for every sexual encounter was advised [25]. The projected figures of ICM inserted in all the 43 PHC facilities were very low during the data review meeting, as shown in Table 1 [26]. The facilities' statistics were ranging between one and two per month. This study, therefore, sought to develop a model to promote the use of ICM in Vhembe District, Limpopo Province.

Table 1

Presents the utilisation of short- and long-acting contraceptive methods in Thulamela and Musina Sub-Districts

\begin{tabular}{|c|c|c|c|c|c|c|c|}
\hline Sub-District & Total PHC facilities & Financial year & Oral pill cycle & Medroxyprogesterone & Norethi-sterone & ICUD & $\begin{array}{l}\text { ICM } \\
\text { Inserted }\end{array}$ \\
\hline \multirow[t]{3}{*}{ Thulamela } & 43 & $2014 / 2015$ & 36171 & 25859 & 49036 & 00 & 4718 \\
\hline & & $2015 / 2016$ & 44472 & 24031 & 43355 & 00 & 1300 \\
\hline & & $2016 / 2017$ & 46830 & 25958 & 42664 & 00 & 110 \\
\hline \multirow{2}{*}{ Musina } & & $2015 / 2016$ & 12771 & 11560 & 10715 & 00 & 349 \\
\hline & & $2016 / 2017$ & 12459 & 9925 & 8641 & 20 & 244 \\
\hline
\end{tabular}

\subsection{Methods}

\subsection{Study Design}

The study was qualitative, explorative, descriptive and contextual to explore SAW's knowledge, practices, and attitudes related to the usage of ICM in Vhembe District of Limpopo Province. The study design was an in-depth emic exploration of SAW views as the researcher had little information about the factors affecting ICM use. The participants gave their views concerning the usage of ICM without being led on what to say, and this gave their emic perspective

\subsection{Study Setting}

The study was conducted in the Vhembe District of Limpopo Province. Vhembe District consisted of four Sub-Districts, Thulamela Sub-District, consisting of 40 clinics and three community health centres ( $\mathrm{CHC}$ ). Makhado Sub-District consisted of 31 clinics and two (02) $\mathrm{CHC}$, and Collins Chabane Sub-District consisted of 30 clinics and three CHC, Musina Sub-District consisted of ten clinics only. All these PHC facilities in Vhembe District render multiple PHC services, including insertion of ICM device as one of the reproductive health services (RHS) or family planning methods, seven days per week; this was informed by the very low numbers of ICM inserted and early removal at all PHC facilities in Vhembe District (OMN and Data Capture's meeting). PHC facilities were purposefully selected in the Vhembe District of Limpopo Province.

\subsection{Study population and sampling}


This study comprises all SAW: Aged 18-45 years who visit the selected PHC facilities for family planning methods and stay in Vhembe District. SAW may be users or non-users of ICM as the method of family planning. A non-probability sampling technique was employed to select PHC facilities with high numbers of RHS statistics. Out of 53 PHC facilities, only 03 CHC in Thulamela Sub-District and 02 PHC facilities Musina SubDistrict were sampled; hence Musina Sub-District does not have CHC.

Non-probability purposive sampling was used to select SAW: Aged 18-45 years, who visit the selected PHC facilities for RHS and stay in Vhembe District. SAW may be users or non-users of ICM as the method of family planning. With the help of the OMN the researchers targeted and briefed the SAW coming for family planning services about the study. After that, SAW were addressed individually to secure informed consent. Those that agreed to participate signed informed consent and confidentiality binding form and then participated in the study. The date for the interview was also agreed upon and SAW the venue for the meeting/ interviews was the PHC facility, a private office allocated by the OMN.

\subsection{Data collection}

Data was collected through five (5) focus group discussions (FGD) using semi-structured interview, which lasted for approximately 45 - 60 minutes. One central question was used to initiate the interview. The interviews were conducted in the language comfortable for each participant, Pedi, Xitsonga and Tshivenda, to ensure their understanding and full participation. The main author conducted the interviews, as she is conversant with those three languages.

The following questions were asked, "What are your perceptions about the usage of ICM? What are the factors that influence utilisation ICM? And what are the factors that influence non-utilisation of ICM?"However, other probing questions were asked as follow-up and paraphrasing to ensure expansion and deepen the discussions to enhance understanding of the phenomenon. Data collection was also aided by using the audio recorder, observations of non-verbal cues and taking of field notes. A total of sixty-two (62) SAW was interviewed and was determined by data saturation.

\subsection{Data Analysis}

Collected data was transcribed verbatim then translated to English using language editors for each language group. Tesch's eight steps of open coding, as outlined in [27] was used to analyse data. The author scrutinised the transcript several times to ensure the identification of the similarities and differences. Data collected was also scrutinised by the co-author before the finalisation of the themes and the sub-themes. Data coding done by the two authors independently. The main author and co-author then discussed their independently formulated themes and subthemes from the coded data and reached a consensus. All the transcripts were scrutinised to ensure that no new codes and themes emerged, and the researchers found that all the quotes in the participant's transcripts support the identified themes. Thus, data saturation was declared.

\subsection{Data quality}

Measures to ensure data quality, such as credibility, dependability, conformability and transferability, were observed. This study achieved credibility by prolonged engagement with the participants during appointments until reaching data saturation. Raw data was transcribed verbatim from interviews; observation notes and recording were kept for scrutiny and verification purpose. The authors collected sufficient detailed descriptions of the data to ensure that the results are a true reflection of why SAW does not use ICM. The data supported this study's results, conclusions, and recommendations as evidence by participants' verbatim quotations.

\subsection{Results}

The results are derived from the interview data with the SAW. Data were collected from December 2018 to May 2019 . Five (5) focus group discussions were conducted with 62 participants. The FGD consisted of 10-15 participants, which ranged between 1 to 15 . Their ages ranged as follows; 22 SAW ages ranged from 18 to 25 years old, 16 SAW ages ranged from 26 to 35 years old and 24 SAW ages ranged from 36 to 45 . The majority of participants (SAW) (29) married, 13 divorced, and 20 were still single. The majority of those SAW were Christians, while only seven were non-Christians. Out of the 62 participants, 12 were permanently employed, 50 participants were not employed, 32 participants passed Grade 12 and 30 had no Grade 12, but 22 managed to complete their tertiary education, and some were already employed. Table 2: shows a summary of the demographic profile of SAW.

Table 1: Presents the utilisation of short- and long-acting contraceptive methods in Thulamela and Musina Sub-Districts 


\begin{tabular}{|c|c|c|c|c|c|c|c|}
\hline Sub-District & Total PHC facilities & Financial year & Oral pill cycle & Medroxyprogesterone & Norethi-sterone & ICUD & $\begin{array}{l}\text { ICM } \\
\text { Inserted }\end{array}$ \\
\hline \multirow[t]{3}{*}{ Thulamela } & 43 & $2014 / 2015$ & 36171 & 25859 & 49036 & 00 & 4718 \\
\hline & & $2015 / 2016$ & 44472 & 24031 & 43355 & 00 & 1300 \\
\hline & & $2016 / 2017$ & 46830 & 25958 & 42664 & 00 & 110 \\
\hline \multirow[t]{3}{*}{ Musina } & 10 & $2014 / 2015$ & 8781 & 7024 & 7747 & 03 & 1243 \\
\hline & & $2015 / 2016$ & 12771 & 11560 & 10715 & 00 & 349 \\
\hline & & $2016 / 2017$ & 12459 & 9925 & 8641 & 20 & 244 \\
\hline
\end{tabular}

Vhembe District DHIS 2014-2017

Table 2

Demographic Profile of Sexually Active Women.

\begin{tabular}{|c|c|c|c|c|c|c|c|c|c|c|c|c|c|}
\hline \multirow{2}{*}{$\begin{array}{l}\text { Participants } \\
\begin{array}{l}\text { Total of } \\
\text { participants }\end{array}\end{array}$} & \multicolumn{3}{|c|}{ Age in years } & \multicolumn{2}{|l|}{$\begin{array}{l}\text { Marital } \\
\text { Status }\end{array}$} & & \multicolumn{2}{|l|}{ Religion } & \multicolumn{2}{|c|}{$\begin{array}{l}\text { Employment } \\
\text { Status }\end{array}$} & \multicolumn{3}{|c|}{ Level of education } \\
\hline & $\begin{array}{l}18- \\
25\end{array}$ & $\begin{array}{l}26- \\
35\end{array}$ & $\begin{array}{l}36- \\
45\end{array}$ & Married & Divorced & Single & Christian & $\begin{array}{l}\text { Non- } \\
\text { Christians }\end{array}$ & Yes & No & $\begin{array}{l}\text { Non- } \\
\text { Grade } \\
12\end{array}$ & $\begin{array}{l}\text { Grade } \\
12\end{array}$ & $\begin{array}{l}\text { Tertiary } \\
\text { education }\end{array}$ \\
\hline 62 & 22 & 16 & 24 & 29 & 13 & 20 & 55 & 07 & 12 & 50 & 30 & 32 & 22 \\
\hline
\end{tabular}

\subsection{Presentation Of Themes And Sub-themes}

Data were transcribed verbatim then translated by the language experts to English from the collected data (for each language). The language experts were sought from the Department of African languages at the University of Venda. Data from SAW yielded one main theme, which is the description of the factors, which contribute to the expected uptake of ICM by SAW, and two sub-themes, which were the following: SAW views about Implant contraceptive method and SAW views about the ICM effectiveness in preventing pregnancy. Table 3: shows one developed theme and two sub-themes from raw data.

Table 3

Description of factors, which contribute to expected uptake of Implant contraceptive method by SAW

\begin{tabular}{|ll|}
\hline Theme & Sub-Themes \\
\hline \begin{tabular}{ll} 
1. Description of the factors which contribute to expected uptake of ICM by & 1.1 SAW views about Implant contraceptive method \\
& $\begin{array}{l}1.2 \text { SAW views about the ICM effectiveness in preventing } \\
\text { pregnancy }\end{array}$ \\
\hline
\end{tabular} \\
\hline
\end{tabular}

\section{Description of the factors which contribute to expected uptake of Implant contraceptive by Sexually active women}

Data from the SAW indicates that most of the women had little knowledge about ICM, making it difficult for them to make informed decisions to utilise the method. It was observed that most of them are interested in the method and would like to use it to enjoy the benefits that they have observed. From this theme to following sub-themes emerged: SAW views about the Implant contraceptive method and SAW views about the ICM effectiveness in preventing pregnancy

Sub-Theme 1.1: Sexually active women's views about Implant contraceptive method

Sexually active women who used this contraceptive method and those who witnessed their friends, peers and relatives using the method commented that it is user-friendly and not user-dependent. Once one is pricked on the inner upper arm of the less active arm, the pain is just felt that day; it will be felt again in three years. Participants commented that ICM is unlike the injectable where you are expected to visit the clinic every two or three months to be injected or collection of supply depending on the type of contraceptive one is using. This was said to be annoying, disturbing and expensive, as one must have transport fees.

They further added that SAW who are on the other contraceptive visit clinics more frequently for review of treatment; meanwhile, those on ICM come once and for all, they visit when there is a need; they visit when they need assistance on the management of side effects, for example, if one is experiencing irregular bleeding. SAW indicated that the issue that ICM causes irregular bleeding is true. Still, in most cases, this is manageable if one follows the prescribed treatment and advice given by professional nurses.

Page 6/12 
Participants clarified that relatives, peers, and friends exaggerate the bleeding, and most of those advocating for these side effects have never used the device. Those interested in utilising the device; who do not want to frequent PHC facilities for review of treatment can manage ICM common side effects. Employed SAW and those studying find the methods very useful and solve their problems as frequent visits to the health facilities interfere with their daily work programs and school activities.

Participants communicated that they are now free of long waiting queues to review their treatment. Participants reported that with ICM there is no need for compliance by the user since the device is not user-dependent. However, those who have not used ICM did not have much information regarding this effective method rather than relying on the information they got while sharing with their peers and friends. Additionally, this device can be utilised by both breastfeeding and non-breast-feeding mothers; as it does not affect milk production nor negatively affects the growth and development of the newborn.

For sexually active women who use ICM, their interest rests on the effectiveness of the method. When ICM device is removed, its medication does not remain in the body like injectables, where some SAW may wait for 9 to 12 months without falling pregnant, especially with medroxyprogesterone. Furthermore, removal of ICM means immediate return to fertility. This was evidence based on what some of the participants confirmed their personal return to fertility after removal of ICM. The device can be removed anytime without the medication remaining in the body like injectables, which remains and lengthens period of returning to fertility.

Sexually active women indicated that some of them experience amenorrhea when utilising ICM, which they feel comfortable of, as some SAW do not like the monthly bleeding process. Their life plans are not interrupted when using this ICM, because unplanned pregnancy is a major lifethreatening problem. This device prevents pregnancy up to $99 \%$ unlike other family planning methods where one can fall pregnant if she forgets to take a pill or return date to be injected. They described how they always feel relaxed when doing their daily duties without thinking of the return date to visit their clinic for review of treatment or not willing to engage in sexual intercourse. The following quotes voiced by participants support these findings:

Participant 1: Female, 34 years, FGD-1

"When using ICM, there is no need to comply with prescribed treatment; in other words, the method is not user-dependent. Once the device is inserted on the inner upper arm, I do not worry about forgetting the time to take or use the medication to prevent the pregnancy. There is no need to set up a telephone reminder to swallow a pill or go to the clinic for injectables or to fetch the monthly supply. Once the device is inserted, and a measured amount of the medication to prevent pregnancy will be released into the bloodstream and start functioning for three years".

Participant 4: Female, 24 years, FGD-1

"I am very comfortable with the method as my menstrual cycle has not changed; although some of my friends say they experienced amenorrhea, however, they are continuing to use the method. You know I have reduced the number of visits to the clinic, as this was costly and timeconsuming. Now I can do my household chores..."

Participant 7: Female, 33 years, FGD-3

"I used it before, and when I wanted to fall pregnant, I just went to the clinic for removal, and after the second month, I was pregnant. Some SAW experience amenorrhea, which they feel comfortable with, as they do not like the bleeding process every month. Their life plans are not interrupted when using this type of $L A R C$, because mistimed pregnancy is not a problem. No need for the frequent return date; they are feeling relaxed when doing their daily duties. Another thing that I like is that I don't have to experience menstruation every month, which is costly as you need to have pads and distressing..."

Participant 9: Female, 23 years, FGD-5

"Implant contraceptive method is one of the best methods I ever came across, the device last for a very long period, which is three years; I think it is user-friendly. We save money meant for transport fees, unlike the other methods that require us to visit facilities more regularly. I think I will also insert the device as it worked well for my sister..."

\section{Sub-Theme 1.2: Sexually active women's views about the effectiveness of Implant contraceptive method in preventing pregnancy}

The implant contraceptive method is one of the LARC, which is highly effective and appropriate for nearly all SAW, including nursing mothers, to delay, space, or limit pregnancies, and they are increasingly popular. During FGD interviews, most participants alluded that ICM is an effective method that prevents unplanned pregnancies, as falling pregnant while using the method is very rare. They indicated that ICM is unlike other methods where one can fall pregnant if one has missed an appointment day to review treatment. There is no risk of falling pregnant once the device is inserted, and one follows the instructions given by the health provider. 
Sexually active women who have never used the device pointed out that ICM seems to be a good method from the information that they got from their peers and friends who used the method. Some of those who have not yet used the method indicated that they are thinking of trying the method to find out for themselves rather than relying on what others tell them. It also shows that SAW is interested in this LARC since they tried to observe and ask others who had experience with this method. SAW who are always busy with their businesses, studies, and other things are not interested in the device's side effects, but they are happy about the effectiveness of this method.

Participant 3: Female, 21 years, FGD-2

"Unexpected pregnancy is not a problem when one is using ICM as a method of pregnancy prevention according to the knowledge I was given at Mutale $\mathrm{CHC}$ when it was introduced to me by the health providers during the launching period. The introducers said it is highly effective more than all the present methods available at health facilities...so I trust them".

Participant 6: Female, 44 years, FGD-1

"I concluded that ICM is a good method; I saw that from a friend of mine. She did not fall pregnant while the device was in her body, which confirms the effectiveness of this method in preventing unplanned pregnancy. When the time for removal was due, once it was removed, she menstruated, then the following month, she missed her period without waiting for a long period. My friend did not have many complaints for three years that I heard people talking about, and I think this is a very good and reliable method".

Participant 5: Female, 22 years, FGD-1

"Yes, sister, hopefully so; I used it when I got married from 2014 to 2017 since I was still finishing my studies and I did not fall pregnant for three years. It worked for me since I learnt about its effectiveness in the prevention of pregnancy. I heard about the rumours, but I felt I must read about the device myself to understand its functions in the human body. This was because I had an interest in this device as it one of the LARC".

\subsection{Discussion}

The unmet contraceptive needs triggered the National Department of Health (NDoH) to introduced Implanon NXT (ICM) in 2014 to increase the range of contraceptive options for SAW, particularly LARC [1]. Despite its low uptake, ICM is viewed as an effective method worldwide, with a low failure rate of $0.05 \%$ compared to other contraceptive methods [28]. In addition, [29] signalled ICM as an effective LARC of fertility regulation helpful to SAW who wish to lengthen their period of contraceptive protection. In contrast, [28] pointed out that oral contraceptives (OC) effectiveness in preventing pregnancy depended on taking it daily and timeously since it is user-dependent. That was also found in this study, where participants indicated the effectiveness of ICM in preventing pregnancy. The other contraceptives were problematic as one needs to remember taking them or going to the health facility after a period to get the injection as a maintenance dose.

Sexually active women indicated that ICM is a safe method for mothers to use when breastfeeding, unlike other OC and injectable contraceptives, which reduces the amount of breast milk production when used. According to [28], hormonal strategies, including combined estrogen-progestin OC, disable breast milk production. In contrast, [30] indicated that contraception with progestin alone, regardless of whether conveyed orally, subdermal or intrauterine, has no malicious impacts on breast milk formation and production or newborn development. While ICM method was said to be good for both non-breastfeeding and breastfeeding moms. [30] Concurred with these findings that manageable side effects are experienced when SAW are utilising this device, and its effectiveness is deemed good for SAW who need to hold up before falling pregnant again.

Participants of this study never mentioned their spouses as people they discuss with them about contraception. It was identified that SAW discusses with their peers or relatives about the different contraceptive methods. This is confirmed by the study conducted by [31], who indicated that the use of ICM does not need the cooperation of a sexual partner as is in the case where partners are using condoms.

It is a concern that SAW do not have sufficient knowledge about ICM compared to other contraceptive methods. This could mean that health providers are not giving more attention to the newly introduced method. It should be clear that for SAW to use the method effectively, they should have more reliable information so that they can make informed decisions. It has been confirmed by [32], who asserted that for effective use of this LARC DoH should consider reskilling, mentoring and supporting health providers to address competency gaps and negative attitudes towards the newly inverted method. Professional nurses are essential to the success or failure of contraceptive programmes, and play a decisive role in shaping how SAW perceives different contraceptive methods.

Advanced interventions are needed to narrow disproportions in contraceptive usage among different population groups and increase LARC users' quality of family planning [33]. ICM is seen as easy to insert as SAW indicated that when they visit the clinic, it does not take time for the device to be inserted in their inner upper arms and is not too painful. SAW identified ICM as being effective in preventing pregnancy with fewer side effects.

A study ICM has several positive features that contribute to its rapid rising popularity which include quickly, safely, and easily inserted in two to three minutes by skilled health providers[34]. ICM is a highly effective contraception for three to five years, as recommended by the studies from [35]. ICM has convenience, LARC very high effectiveness, ease of provision, and speedy return to fertility upon removal. 
Implant contraceptive method failure is below $1 \%$ compared to OC $6 \%$ and injectables is about $9 \%$. Nevertheless, [34] revealed that the use of injectables remained high and rising but has declined in nine of 12 countries during their study in Africa. ICM use does not necessitate pelvic examination or abdominal surgery like Intra-uterine copper devices (IUCD) and female sterilisation. Ready reversibility: No further routine action is necessary until the client wants ICM removed. The removal of the device is usually a speedy and straightforward procedure lasting for three to seven minutes, even though a few removals may be difficult and possibly necessitating referral. Generally, ICM have high client satisfaction, as implied in their high continuation rates [34].

\subsection{Conclusion}

The objectives were to explore the views of SAW about the usage of ICM, identify the factors that influence the utilisation and non-utilisation of ICM by SAW in Vhembe District. The research revealed that the launching of this newly inverted method did not cover all areas served by Vhembe District. Professional nurses and OMN should inform public members about newly introduced RHS at PHC facilities to enhance usage. That may be done through health education, mass campaigns and individuals, couples or family counselling about the new method.

\subsection{Recommendations}

Recommendations were formulated based on the findings of the current study. The authors made recommendations related to nursing practice and further nursing research.

\subsection{Nursing Practice}

- For this service to run smoothly at PHC facilities, Vhembe District responsible managers to support professional nurses and OMN who are hands on rendering RHS. Introduction of new RHS such ICM requires effective interventions by District managers, through training all (community health workers) $\mathrm{CHW}$, professional nurses and OMN working at PHC facilities. CHW to continue giving information in the communities and refer SAW, partners and collaborates to health facilities for services. During outreach services, public members to be encouraged to visit PHC facilities for more information and questions regarding ICM.

- Involvement of all stakeholders (churches, women's groups, clinic committee members and ward counsellor's meetings) in the communities during launching campaigns may facilitate the use of ICM.

- Mobile clinic staff to be trained to insert ICM at visiting points far from PHC facilities to avail the service.

- Professional nurses to encourage SAW partners and family members to support them in their childbearing ages, to visit clinics/PHC facilities for use of LARC.

\subsection{Nursing Research}

- Challenges faced by SAW in childbearing ages regarding newly introduced ICM need further investigation.

- Challenges of male sexual partners regarding giving support to their female partners is critical and need to be dealt with.

\section{Abbreviations}

$\mathrm{CHC}$

Community health centers

$\mathrm{CHW}$

Community health workers

$\mathrm{DoH}$

Department of Health

FGD

Focus group discussion

HIV/AIDS

Human Immunodeficiency Virus/Acquired Immunodeficiency Syndrome

ICM

Implant contraceptive method

ICUD

Intra-uterine copper devices

LARC

Long acting contraceptive method

$\mathrm{NDoH}$

National Department of Health 
OC

Oral contraceptives

OMN

Operational managers

$\mathrm{PHC}$

Primary health care

RHS

Reproductive health services

SACM

Short acting contraceptive methods

SAW

Sexually active women

STIs

Sexual transmitted infections

TOP

Termination of pregnancy

US

United States

\section{Declarations}

\subsection{Ethical Approval}

The authors ensured compliance with ethical standards to protect the rights and confidentiality of the participants. Approval for this study was obtained from the University of Venda Research Ethics Committee (SHS/19PDC/03/1503), and Limpopo Department of Health Research Ethics. Participants were fully informed about the study and not forced to participate as voluntary participation was ensured by allowing them to withdraw at any time without any penalties. Informed consents were obtained before the beginning of the interview, and these signed consent forms were kept separately from the transcript to ensure anonymity.

\subsection{Consent for publication}

Not Applicable

\subsection{Availability of data and material}

Data is available upon request

\subsection{Competing Interests}

The authors declare that they have no competing interests.

\subsection{Funding}

The research was funded by the The University of Venda, Research Directorate, funded this this research. The funder provided resources to cover data collection, management and analysis. Researchers wrote and submitted reports to appraise the funder of progress. The funder's role was to provide resources to carry out this research successfully.

\subsection{Authors' Contributions}

RCS was a PhD in Public Health student at the University of Venda during the time this research was conducted. The author conceptualised the research as partial fulfilment of the requirements for the PhD qualification. RTL was the Supervisor, while MSM was the Co-Supervisor and the two contributed by guiding the study in conceptualising the research idea, carrying out the research and preparation and submission of the manuscript. All authors read and approved the final paper.

\subsection{Acknowledgements}

Not Applicable

7.8 Authors' Information 
RCS was a PhD in Public Health student at the University of Venda in South Africa. RTL is a Research Professor at the School of Health Sciences at the University of Venda in Thohoyandou in South Africa.MSM is a Professor and Lecturer in the Deaprtment of nursing at the same institution.

\section{References}

1. Bracken J, Graham CA. "Young women's attitudes towards, and experiences of, long-acting reversible contraceptives". The European Journal of Contraception Reproductive Health Care. 2014;19:276-84.

2. Russo JA, Miller E, Gold MA. "Myths and misconceptions about long-acting reversible contraception (LARC)". J Adolesc Health. 2013;52:1421.

3. Dansereau E, Schaefer A, Hernández B, Nelson J, Palmisano E, Ríos-Zertuche D, Woldeab A, Zúñiga MP, Iriarte EM, Mokdad AH, Bcheraoui C. "Perceptions of and barriers to family planning services in the poorest regions of Chiapas, Mexico: a qualitative study of men, women, and adolescents," Reproductive health, 14(1), p.129, 2017.

4. Stephenson R, Vwalika B, Greenberg L, Ahmed Y, Vwalika C, Chomba E, Kilembe W, Tichacek A, Allen S. "A randomised controlled trial to promote long-term contraceptive use among HIV-serodiscordant and concordant positive couples in Zambia". Journal of Women's Health. 2011;20:567-74.

5. Eliason S, Baiden F, Quansah-Asare G, Graham-Hayfron Y, Bonsu D, Phillips J, Folch-Lyon E, de la Macorra L, Schearer SB, "Focus group and survey research on family planning in Mexico," Studies in Family Planning, pp.409-432, 2013.

6. Ramathuba DU, Khoza IB, Netshikweta ML. "Knowledge, attitudes and practice of secondary school girls towards contraception in Limpopo Province". Curationis. 2012;35,:1-7.

7. Gebremariam A, Addissie A. "Intention to use long acting and permanent contraceptive methods and factors affecting it among married women in Adigrat town, Tigray, Northern Ethiopia". Reproductive health. 2014;11:24.

8. Mekonnen G, Enquselassie F, Tesfaye G, Semahegn A. "Prevalence and factors affecting use of long acting and permanent contraceptive methods in Jinka town, Southern Ethiopia: a cross sectional study" Pan African Medical Journal, 18, 2014.

9. Jacobstein R, Stanely H. "Contraceptive implants: providing better choice to meet growing family planning demand". Global Health: Science Practice. 2013;1,:11-7.

10. Keesara SR, Juma PA, Harper CC. "Why do women choose private over public facilities for family planning services? A qualitative study of post-partum women in an informal urban settlement in Kenya". BMC Health Serv Res. 2015;15:335.

11. Kipps S. "Prescribing contraception safely and effectively". Nurse Prescribing. 2014;12,:296-301.

12. Mhlanga R, Molefe N, Fawcus S, Pattison R, Rout C, Kunene B, Chweneyagae D, Farina Z, Moran N, Khaole. "The impact of HIV infection on maternal deaths in South Africa". South African Journal of Obstetrics Gynaecology. 2014;18:70-6.

13. Carr S, Espey E. "Intrauterine devices and pelvic inflammatory disease among adolescents". J Adolesc Health. 2013;52:22-8.

14. Nsubuga H, Sekandi JN, Sempeera H, Makumbi FE. Contraceptive use, knowledge, attitude, perceptions and sexual behavior among female University students in Uganda: a cross-sectional survey. BMC Womens Health. 2015;16:6.

15. Cea Soriano L, Wallander MA, Andersson S, Filonenko A. \& L.A. García Rodríguez "Use of long-acting reversible contraceptives in the UK from 2004 to 2010: analysis using the health improvement network database". The European Journal of Contraception Reproductive Health Care. 2014;19:439-47.

16. Rowlands S, Searle S. "Contraceptive implants: current perspectives". Open Access Journal of Contraception. 2014;5,:73-84.

17. Alemayehu M, Kalayu A, Desta A, Gebremichael H, Hagos T, Yebyo H. "Rural women are more likely to use long acting contraceptive in Tigray region, Northern Ethiopia: a comparative community-based cross sectional study". BMC Womens Health. 2015;15:71.

18. Joseph EJ, "Utilisation of family planning services among sexually active people living with HIV/AIDS in Taso Tororo," Makerere University 2010.

19. Kimani J, Warren CE, Abuya T, Ndwiga C, Mayhew S, Vassall A, Mutemwa R, Askew I. "Use of HIV counseling and testing and family planning services among postpartum women in Kenya: a multicentre, non-randomised trial". BMC Womens Health. 2015;15:104.

20. Anguzu R, Tweheyo R, Sekandi NJ, Zalwango V, Muhumuza C, Tusiime S, Serwadda D. "Knowledge and attitudes towards use of long acting reversible contraceptives among women of reproductive age in Lubaga division, Kampala district, Uganda". BMC Res Notes. 2014;7:153.

21. Nishtar NA, Sami N, Alim S, Pradhan N. "Determinants of contraceptives use amongst youth: an exploratory study with family planning service providers in Karachi Pakistan". Global journal of health science. 2013;5:1.

22. Conversation with OMN. 2017.

23. Vhembe, District. District Health Information System 2014/2015, 2015/2016, 2016/2017.

24. Lakha F. "Young women's experiences of side effects from contraceptive implants: a challenge to bodily control: "I think it depends on the body, with mine it didn't work":. explaining young women's contraceptive implant removal; 2013.

Page $11 / 12$ 
25. Stewart M, Bateson D. "Choosing non-oral, long-acting reversible contraception. " Australian prescriber. 2016;39:153.

26. PHC Facilities Input Forms, 2017.

27. Creswell JW. \& J.D. Creswell "Research design: Qualitative, quantitative, and mixed methods approaches. "Sage publications; 2017.

28. Mkansi MA, "Knowledge, attitudes and perceptions of secondary school going girls towards Implanon contraceptive at Bokamoso Secondary School, Polokwane District Municipality, Limpopo Province, South Africa (Doctoral dissertation)," 2018.

29. Tekelab T, Sufa A, Wirtu D. "Factors affecting intention to use long acting and permanent contraceptive methods among married women reproductive age groups in Western Ethiopia: a community based cross sectional study". Fam Med Sci Res. $2015 ; 4: 2$.

30. Javed N, Mehmood T, Almas H. "Experiences of Women and Assessment of Efficacy and Side Effects of Sub-Dermal Implants in Rural Islamabad" Pakistan Journal of Medical Research, 55(4), 2016.

31. Agboghoroma CO. Contraception in the Context of HIV/AIDS: A Review. African Journal of Reproductive Health. 2011;15,:15-24.

32. Adeagbo OA, Mullick S, Pillay D, Chersich MF, Morroni C, Naidoo N, Pleaner M, Rees H. "Uptake and early removals of Implanon NXT in South Africa: Perceptions and attitudes of healthcare workers" South African Medical Journal, 107(10), 2017.

33. Medhanyie AA, Desta A, Alemayehu M, Gebrehiwot T, Abraha TA, Abrha A, Godefay H. "Factors associated with contraceptive use in Tigray, North Ethiopia". Reproductive health. 2017;14:27.

34. Jacobstein R. "Liftoff: the blossoming of contraceptive implant use in Africa". Global Health: Science Practice. 2018;6(1):17-39.

35. WHO/DRHR and Johns Hopkins Bloomberg School of Public Health Center for Communication Programs (CCP), "Family planning: a global handbook for providers, 2011 update". Baltimore and Geneva: CCP and WHO; 2011. Available from: http://www.fphandbook.org/. 